\title{
Propuesta de Implementación de Resultados de Aprendizaje en la Institución Universitaria Digital de Antioquia
}

Londoño-Ciro, Libardo-Antonio ${ }^{1 *}$; ; De La Rosa-Isaza, Jasson Alberto';

Gutiérrez-Ángel, Catalina María'; Benjumea-Garcés, Juan Sebastián'

IInstitución Universitaria Digital de Antioquia, Vicerrectoría Académica, Medellín, Colombia

\section{RESUMEN}

En este artículo se propone un modelo que contempla unas fases de desarrollo, para la implementación de los resultados de aprendizaje en la Institución Universitaria Digital de Antioquia. Se presentan algunas bases conceptuales provenientes de autores que han escrito sobre el tema y consideraciones al respecto del Ministerio de Educación Nacional de Colombia. Estas bases conceptuales se analizan con el fin de determinar la viabilidad de la implementación del modelo, identificando sus ventajas potenciales, los mecanismos de aplicación y sus alcances.

Palabras clave: resultados de aprendizaje; gestión curricular; educación virtual.

\section{Implementation Proposal of Learning Outcomes at Digital University Institution of Antioquia}

\section{ABSTRACT}

This article proposes a model which contains some stages of development, for the implementation of learning results in the Digital University Institution of Antioquia. Some conceptual bases are shown from authors who have written about it and considerations in this regard made by the Ministry of National Education of Colombia. These conceptual bases are analyzed in order to determine the viability of the model implementation, identifying its potential advantages, the application strategies and its purposes.

Keywords: learning results; curriculum management; virtual education 


\section{INTRODUCCIÓN}

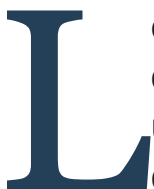

os resultados de aprendizaje son declaraciones expresas sobre lo que se espera que un estudiante conozca y esté en condiciones de demostrar de lo que ha estudiado desde el momento en que comienza hasta que finaliza su formación. Este concepto implica para el sector educativo nuevos retos, ya que el proceso de enseñanza - aprendizaje se centra fundamentalmente en el aprendizaje $y$, por lo tanto, las metodologías activas y la evaluación orientadas a los estilos de aprendizaje cobran mucha relevancia en el proceso. Según el enfoque de aprendizaje del Decreto 1330 de julio de 2019, los educadores y educandos deben evidenciar en la práctica las destrezas y las habilidades adquiridas en el proceso formativo, lo cual representa un avance en materia de legislación educativa para nuestro país.

En este artículo se propone un modelo para la implementación de los resultados de aprendizaje en la IU Digital de Antioquia y sus estrategias educativas. Se plantea un marco teórico que sirve como sustento y antecedente de esta propuesta, se plantean los componentes del modelo, la discusión, conclusiones y las prospectivas a mediano plazo.

\section{METODOLOGÍA}

Según el Decreto 1330 del Ministerio de Educación Nacional de julio 25 de 2019, "los resultados de aprendizaje son concebidos como las declaraciones expresas de lo que se espera que un estudiante conozca y demuestre en el momento de completar su programa académico". Ese planteamiento es similar al considerado en el Proceso de Bolonia, en donde un resultado de aprendizaje se define como "una declaración de lo que el estudiante se espera que conozca, comprenda y sea capaz de hacer al finalizar un periodo de aprendizaje" (Proceso Bolonia, 1999). El Marco Europeo de Cualificaciones para el aprendizaje permanente (EQF-MEC, 2008) señala que los resultados de aprendizaje tienen por fin último "fomentar procesos de aprendizaje permanente para la creación de la sociedad del conocimiento" y, para su implementación, se deben hacer adaptaciones curriculares, tecnológicas y reformas financieras. Esta enunciación sobre los resultados de aprendizaje y los requisitos que implica su implementación han propiciado muchas objeciones en Europa, que van desde críticas al mejoramiento de la enseñanza universitaria, hasta otras que señalan que alrededor de las reformas se pretende una progresiva política de mercantilización del ámbito universitario (Gibbs, 2008).

Los resultados de aprendizaje se pueden clasificar en tres categorías (Olga Zlatkin et al, 2018):

- Conocimientos (teóricos y/o fácticos).

- Destrezas cognitivas frente al uso del pensamiento lógico, intuitivo y creativo; y prácticas frente al uso de métodos, materiales, herramientas e instrumentos.

- Competencia: responsabilidad y autonomía.

Según Abuaiadah (2018), los resultados de aprendizaje son diferentes de los objetivos de enseñanza temática de una asignatura y están relacionados con lo que se espera que el estudiante conozca (el cómo), comprenda (el porqué) y sea capaz de hacer (el para qué) al terminar una asignatura y un programa de formación. Abuaiadah sugiere que los resultados de aprendizaje deben estar correlacionados con estrategias concretas de enseñanza y de evaluación; tales como:

- Una enseñanza práctica que permita la participación del alumno a través de ejercicios, trabajo en grupo, prácticas profesionales, etc.

- Una evaluación continua en lugar de una evaluación parcial, lo cual implica un seguimiento diario al trabajo del alumno a través del uso de las TIC y tutorías personalizadas.

En la comunidad académica se suelen confundir los resultados de aprendizajes con el desarrollo de competencias, debido a que la frontera entre los resultados de aprendizaje y el desarrollo de competencias es difusa:

- Para el EQF-MEC, "las competencias son parte de los resultados del aprendizaje".

- Para el proyecto Tuning, las competencias se dividen en específicas y genéricas, e incluyen "conocimientos y comprensión, saber cómo actuar y saber cómo ser"; por lo tanto, "los resultados del aprendizaje expresan el nivel de competencia adquirido".

- Para el Marco de Cualificaciones del Espacio Europeo de Educación Superior (EEES, 2005), los resultados del aprendizaje son producto del proceso de enseñanza, "el término competencias está incluido en el concepto de resultados del aprendizaje".

- Para la Agencia Nacional de Evaluación de la 
Calidad y Acreditación española (ANECA, 2014), "los resultados del aprendizaje son concreciones de las competencias para un determinado nivel y que son el resultado del proceso de enseñanza-aprendizaje" de los estudiantes. Por lo tanto, "los resultados del aprendizaje describen lo que los estudiantes deben ser capaces de hacer al término del proceso formativo o de la asignatura", es decir, deben definirse con claridad, ser observables y evaluables, ser factibles y alcanzables por los estudiantes.

Basado en lo anterior, es fundamental que las instituciones, en concordancia con su identidad institucional, definan un contexto de referencia desde el cual aborden la conceptualización y la puesta en práctica de los resultados de aprendizaje en sus procesos de formación.

Algunos autores sugieren que para la definición de los resultados de aprendizaje se tengan en cuenta las siguientes consideraciones (Yepes, 2017):

- Antes de enumerar los resultados del aprendizaje, incluir frases como "al terminar con éxito esta asignatura, los estudiantes serán capaces de...".

- Comenzar con un verbo de acción seguido del objeto del verbo (¿qué es lo hecho?), del contexto (¿en qué área o contexto se puede desarrollar la acción?) y la finalidad (¿para qué es útil lo definido en el objeto del verbo?). Ejemplo: Utilizar (verbo de acción) conceptos y herramientas (objeto de la acción) de la regulación nacional sobre salud (contexto) para la elaboración de propuestas de intervención y mejora de procesos y procedimientos institucionales (finalidad).

- Considerar resultados del aprendizaje que incluyan los planos cognitivo, subjetivo y psicomotor.

- Incluir solo resultados del aprendizaje que el estudiante sea capaz de alcanzar.

- Los resultados de aprendizaje deben ser coherentes con el nivel de formación.

- La redacción de los resultados de aprendizaje debe ser clara y comprensible para otros profesores, los estudiantes y la sociedad en general.

- Se debe definir cómo se pueden medir y evaluar los resultados de aprendizaje (indicadores).

- Se deben definir los requerimientos conceptuales y tecnológicos que se requieren para que los resultados de aprendizaje sean alcanzables.

- Los procesos de evaluación deben ser coherentes con la posibilidad alcanzar los resultados de aprendizaje.

\section{Propuesta}

La propuesta planteada para la implementación de los resultados de aprendizaje en la IUDigital, tiene 3 fases:

Fase 1. Resultados de aprendizaje e identidad institucional. En primer lugar, la Institución debe declarar en qué contexto conceptual va a entender e implementar los resultados de aprendizaje. Esta declaración debe ser coherente con la naturaleza institucional, es decir, si la institución es de docencia, investigación o docencia con investigación, los resultados de aprendizaje deben ser distintos.

En el contexto de su misión institucional, la IU Digital tiene una naturaleza formativa, por lo cual se entienden los resultados de aprendizaje como aquello que los estudiantes serán capaces de hacer o transformar con base en lo que fueron capaces de conocer (el qué), mediante metodologías activas que promuevan el aprendizaje (el cómo) orientados a un contexto de transformación social a nivel de los propósitos de formación de los programas y su interacción con las áreas de aprendizaje (el para qué).

Los resultados de aprendizaje deben definirse a nivel de programa y de asignaturas o de áreas, teniendo en cuenta que:

- La definición del resultado de aprendizaje del programa debe estar en función de las destrezas y las actitudes fundamentales de los egresados en el contexto de una disciplina específica.

- La definición de los resultados de aprendizaje de las asignaturas o las áreas de conocimiento debe estar en función del qué, el cómo y el para qué, y debe contribuir al logro de los resultados del aprendizaje del programa.

Fase 2. Enfoque de desarrollo y evaluación de los resultados de aprendizaje. Es preciso hacer una reflexión de la gestión curricular que permita repensar los enfoques metodológicos de enseñanza - aprendizaje y el proceso evaluativo de la Institución, de manera que los resultados de aprendizaje se puedan desarrollar y medir. En la Figura 1 se propone un modelo para el desarrollo metodológico y la evaluación de resultados de aprendizaje en la IU Digital. 
Figura 1. Modelo de desarrollo metodológico y evaluación de resultados de aprendizaje.

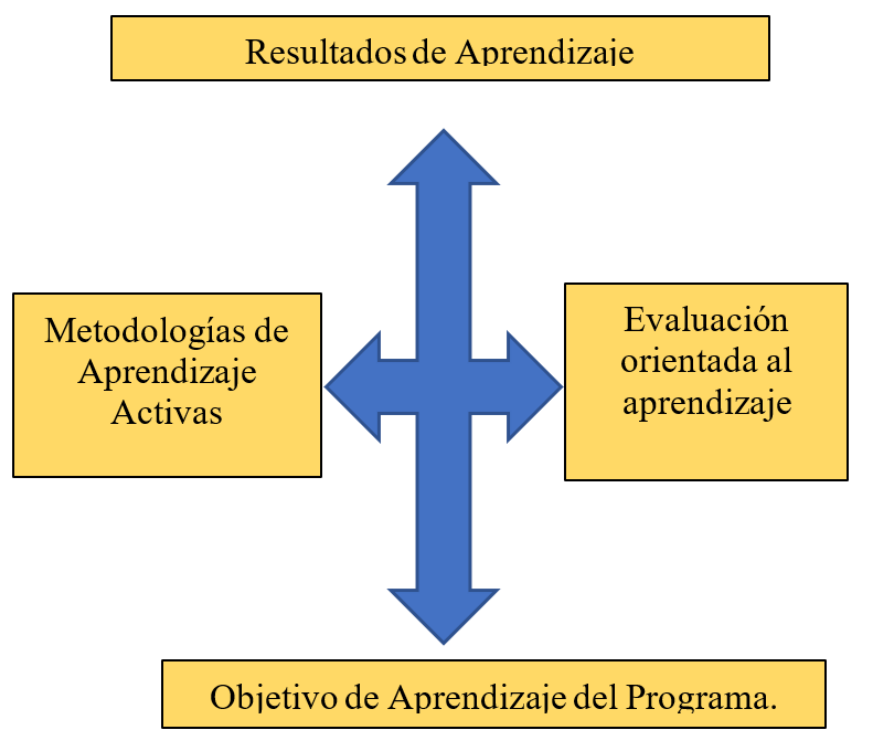

Nota: Fuente: Elaboración propia (2020).

Los componentes del modelo de la Figura 1 se describen a continuación:

- Metodologías de Aprendizaje Activas. Es necesaria una correlación hologramática entre las metodologías de enseñanza - aprendizaje de las asignaturas, los objetivos de aprendizaje de los programas, y los resultados de aprendizaje. La implementación de metodologías activas puede servir a este propósito. En general, la ruta para el diseño y desarrollo de asignaturas debe tener en cuenta las consideraciones del flujograma mostrado en la Fig. 2.
Figura 2. Flujograma de diseño y desarrollo de asignaturas. Adaptado de ANECA (2014).

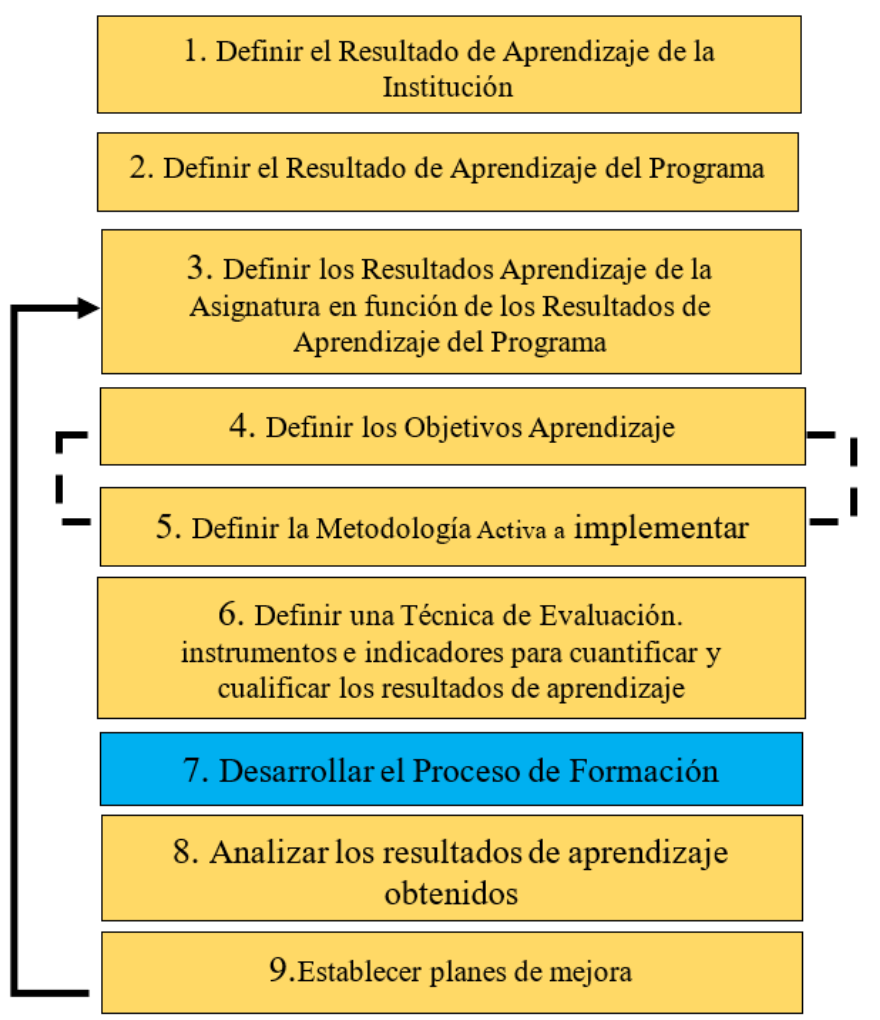

Nota: Fuente: Adaptación propia (2020).

- Evaluación orientada al aprendizaje. Si la evaluación se orienta al aprendizaje y se desarrolla como un proceso continuo, con el acompañamiento de los docentes, entonces la evaluación será un momento más del aprendizaje. Esto implica el diseño de instrumentos e indicadores que permitan cuantificar y cualificar los resultados de aprendizaje.

- Objetivo de Aprendizaje del Programa. Implica una definición de los resultados de aprendizaje del programa en función de las destrezas y las actitudes fundamentales de los egresados en el contexto de una disciplina específica.

Fase 3. Ruta crítica para la implementación de resultados de aprendizaje. Para la implementación de la propuesta, la IU Digital debe resolver una serie de desafíos:

- Formular los resultados de aprendizaje de los programas, las áreas de conocimiento y las asignaturas.

- Hacer una gestión curricular que reformule los aspectos generales para la construcción de planes de estudio en función de resultados de aprendizaje, metodologías activas de apren- 
dizaje y procesos de evaluación. Esto implica pensar en la agrupación de asignaturas por resultados de aprendizaje y una revisión del concepto de proyecto integrador.

- La operacionalización de procedimientos, diseño de instrumentos y formatos, para la elaboración de cartas descriptivas de las asignaturas.

- Una revisión de los planes de mejoramiento académico en función del concepto de resultados de aprendizaje.

\section{RESULTADOS Y/O DISCUSIÓN}

Crown (2019) establece que en la modalidad de educación a distancia, mediada por herramientas digitales y virtuales en las que se implementan procesos de aprendizaje automático, es natural la implementación de herramientas en línea para la obtención de información para la clasificación y estimación de variables educativas que puedan dar cuenta del desarrollo y evaluación de los resultados de aprendizaje. Sin embargo, el uso del aprendizaje automático para la inferencia causal todavía está evolucionando, lo que hace que se tengan divergencias marcadas como las planteadas por Khodabandelou y Daud (2015), quienes postulan que la presencialidad es esencial para el desarrollo y evaluación de los resultados de aprendizaje. Cabe anotar que el crecimiento exponencial de las Tecnologías de la Información y la Comunicación, las relaciones humanas y la educación, tienden a ubicarse cada vez más en la virtualidad, por lo que, con las aplicaciones y tecnologías diseñadas para estos fines, resulta totalmente factible orientar el desarrollo y la medición de resultados de aprendizaje con el respaldo de la virtualidad. En este sentido, el modelo propuesto permitiría el desarrollo de resultados de aprendizaje en modalidad virtual.

La propuesta es pertinente en la medida en que las instituciones educativas colombianas han comenzado a aplicar metodologías para el desarrollo y evaluación de resultados de aprendizaje en el marco de la nueva normativa de condiciones de calidad, expedida por el Ministerio de Educación Nacional de Colombia. Y es original y novedosa por tratarse de una propuesta de implementación en la modalidad virtual que caracteriza a la IU Digital.

\section{CONCLUSIONES}

Se propone un modelo para la implementación de los resultados de aprendizaje con tres fases de desarrollo. El modelo plantea una serie de desafíos institucionales que, a la vez, establece rutas de reflexión y de acción en torno a los resultados de aprendizaje:

- La institución debe definir un contexto conceptual en el que los resultados de aprendizaje y la identidad institucional estén en sincronía.

- La institución debe hacer una gestión curricular coherente con la implementación, el desarrollo y la evaluación de los resultados de aprendizaje.

- La institución debe hacer una gestión académica y financiera acorde con la implementación, el desarrollo y la evaluación de los resultados de aprendizaje.

\section{Prospectiva}

El modelo propuesto presenta para la IU Digital las siguientes ventajas en el corto y el mediano plazo:

- Posibilita la transversalización de contenidos, áreas de conocimiento, asignaturas y proyectos integradores.

- Fortalece el diseño de los OVA en función de los resultados de aprendizaje.

- Fortalece el vínculo docente - estudiante al mediar entre ambos el propósito de desarrollar y validar los resultados de aprendizaje.

- Fortalece la gestión curricular basada en el aprendizaje. 


\section{REFERENCIAS}

Abuaiadah, D., Burrell, C., Bosu, M., Joyce1, S., \& Hajmoosaei, A., (2018). Assessing Learning Outcomes Of course Descriptors. Containing Object Oriented Programming Concepts. New Zealand Journal of Educational Studies. https://doi.org/10.1007/ s40841-019-00139-y

ANECA (2014). Guía de apoyo para la redacción, puesta en práctica y evaluación de los resultados del aprendizaje. www.aneca.es/Documentos-y-publicaciones/Otras-guias-y-documentos-de-evaluacion/ Guia-de-apoyo-para-la-redaccion-puesta-en-practica-y-evaluacion-de-los-RESULTADOS-DEL-APRENDIZAJE.

Crown, W. (2019). Real-World Evidence, Causal Inference, and Machine Learning. Value in Health, 22(5), 587-592. https://doi.10.1016/j.jval.2019.03.001.

EEES (2005). Marcos de cualificaciones en el Espacio Europeo de Educación Superior. www.eees.es/es/ eees-bolonia-hacia-la-convergencia-marcos-calificaciones

Eraut, M. (2006). Aprendizajes y competencias: enfoques que los relacionan en Educación Superior. Seminario RED-U. Universidad de Barcelona.

EQF-MEC (2008). Marco Europeo de Cualificaciones para el aprendizaje permanente. https://web.archive. org/web/20100617004512/http://crue.org/export/sites/Crue/procbolonia/documentos/doccomisioneuropea/Marco_cualificaciones_EQF_ MEC_2008.pdf.

Gibbs, G. (2008). Bolonia no mejora la calidad universitaria. El Economista.es. https://ecodiario.eleconomista.es/educacion/noticias/634124/07/08/ Bolonia-no-mejorara-la-calidad-universitaria-segun-un-profesor-de-Oxford.html

Khodabandelou, R., Jalil, H. A., Ali, W. Z. W., \& Daud, S. M. (2015). Presence and Perceived Learning in Different Higher Education Blended Learning Environments. International Journal of Mobile and Blended Learning, 7(3); 59-70. https://doi.10.4018/ ijmbl.2015070104.

Moreno-Olivos, T. (2010). El currículo por competencias en la universidad: más ruido que nueces. Revista de la educación superior. 39(154); 77-90. www. scielo.org.mx/scielo.php?script=sci_arttext\&pi$\mathrm{d}=$ S0185-27602010000200004\&lng=es\&tlng=es.

Ruiz, J., \& Moya, S. (2018). Evaluación de las competencias y los resultados de aprendizaje en destrezas y habilidades en los estudiantes de Grado de Podología de la Universidad de Barcelona. Educación Médica. 21(2);127-136. https://doi.org/10.1016/j.edumed.2018.08.007 https://doi.org/10.47185/27113760.v1n1.5

Yepes, V. (2017). Qué son los resultados de aprendizaje en el ámbito universitario. Universitat Politècnica de València. https://victoryepes.blogs.upv. es/2017/12/19/resultados-aprendizaje/

Zlatkin-Troitschanskaia, O; et al. (2018). Assessment of Learning Outcomes in Higher Education. Springer International Publishing. https://doi. org/10.1007/978-3-319-74338-7 